\title{
Energy for Safety Information Transmitted in Safety Control System*
}

\author{
Noboru SUGIMOTO** and Koichi FUTSUHARA***
}

\begin{abstract}
The safety control system, which is defined as machine operation controlled according to safety confirmation information, is explained as an interlocking model that functions so that energy output from the machine is permitted only while the information (safety information) reports safety. The information transmission properties presented in this model are applied to all devices in this interlocking system, including the sensors for producing safety information and the devices for transmitting/outputting energy. A small energy-level signal signifying safety is normally produced in a pickup element in the sensor. Enormous power generated by amplifying the energy of the safety signal is supplied to the machine for use in its powerful operation. This paper discusses the condition of the energy transmission process in which energy is produced in a safety sensor as a signal indicating safety and is amplified in the interlocking system up to the sufficient level for performance of machine duties. Firstly, in this paper, the characteristics of information to be provided with the means of information processing in the interlocking system are clarified. Secondly, a logical fail-safe model is proposed for clarifying the production condition of the safety information. Lastly, this fail-safe energy-transmitting system with the safety information can be applied to not only electrically but mechanically processed safety information.
\end{abstract}

Key Words : Mechatronics, Robotics, Safety, Safety System, Safety Control, Safety Control System, Interlocking, Fail-Safe

\section{Introduction}

The authors have already defined safety control ${ }^{(1)}$ as the mechanical operation in which hazardous work is performed based on the condition of safety. Furthermore they have described the basic construction of a safety control system by an interlock model in which energy is output based on the permission of safetysignifying information (safety information) ${ }^{(2)}$. The information transmission characteristics represented by the interlock model were shown to be commonly applicable to both the sensor system that samples the safety information and the energy transmission sys. tem to which the safety information is directly transmitted ${ }^{(3)}$.

Normally, safety-signifying information is input to the sensors at small energy. Amplification is neces-

* Received 22nd July, 1991. Paper No. 89-1526 A

* Mechanical Safety Division, Research Institute of Industrial Safety, Ministry of Labour, 1-4-6 Umezono, Kiyose-shi, Tokyo 204, Japan

*** Research \& Development Center, Nippon Signal Co. Ltd., 1-13-8, Kamikizaki, Urawa-shi, Saitama 338, Japan sary for producing high-output energy for hazardous work to be performed based on the safety signifying information. This paper discusses the characteristics of such an energy amplification process in the interlock system that the safety information is sampled and hazardous work is actually performed. Section 2 describes the required characteristics of the information processing system in which safety information is generated and output energy is produced based on the safety information. Section 3 presents, by a logic model, the conditions under which safety information is generated in a simple work machine with an external energy source. Section 4 demonstrates the principle of construction of a concrete fail-safe computing system by utilizing the logic model. Section 5 demonstrates that fail-safe information transmission is performed by common electrically and mechanically schemes.

\section{Safety Confirmation and Output Energy Generation Process}

\section{1 Safety confirmation process}

Safety is understood as a concept opposed to that of hazard. In other words, safety is a concept that 
occurs in the process in which hazard is recognized, predicted, and avoided. Safety is confirmed, based on the following two principles:

( i ) Safety (no accident) and hazard (accident) cannot occur in the same place at the same time.

(ii) Hazard (accident) can be predicted, but the prediction involves error. If the error (or uncertainty) is regarded as hazard, safety can be ensured.

Principle ( i ) means that true safety (no accident) and hazard (accident) are negatively related. Safety we predict, however, is not simple nonaccident as opposed to accident and is safety at least not leading to an accident. Suppose that safety and hazard are denoted by logic 1 and 0 , respectively, and that safety given by principle ( $\mathrm{i}$ ) and safety predicted by principle (ii) are denoted by the binary logic variables $S_{c}(t) \in\{1,0\}$ and $S_{e}(t) \in\{1,0\}$, respectively. Then the following equation must hold as such a logic relation between safety $S_{c}(t)$ and safety $S_{e}(t)$ that when there is hazard $\left[S_{c}(t)=0\right]$, safety $\left[S_{e}(t)=1\right]$ must not be erroneously predicted:

$$
\forall t, S_{c}(t) \geq S_{e}(t) \text {. }
$$

Here is considered an energy transmission system that produces output, based on safety predicted as expressed by Eq. ( 1 ).

\subsection{Generation process of output energy based on safety confirmation}

The dynamic energy outputted in hazardous work is produced when it is safe and is not produced when it is hazardous. If $u$ is output energy in the safe state and $u^{\prime}$ is energy in the hazardous state, the following inequality holds :

$$
u>u^{\prime}
$$

The error characteristics of the energy $u$ and energy $u^{\prime}$ expressed by Eq. ( 2 ) may be considered for present manufacturing machines and transportation machines as follows:

$u$ : Normally, the output state $u$ is allowed to erroneously change to $u^{\prime}$ as is the case with the movable parts of trains, automobiles, and robots. The erroneous change to the output state $u^{\prime}$ is not allowed for the movable parts of aircraft flying above, trains not permitted to suddenly stop, and robots not allowed to drop workpieces. In principle, therefore, no error is allowed for the output state $u$.

$u^{\prime}$ : No error is allowed in the output state that indicates $u^{\prime}$, but error is normally allowed under the condition where the output $u^{\prime}$ is not produced.

If the aforementioned output energy is denoted by the binary logic variable $U$ (logic 1 for the level of the energy $u$ and logic 0 for the level of the energy $u^{\prime}$ ), the output $U=1$ is the energy outputted based on the confirmation of safety, and this safety confirmation is the predicted safety $S_{e}(t)=1$ in Eq. (1). The means $F$ is thus required for transmitting the safety confirmation signal and producing the output energy between the confirmation of safety and the production of the output energy. The means $F$ is called the information transmitting means here. If the function of the means $F$ is denoted by the logic variable $F\left(S_{e}\right)$ and if the transmission of safety-signifying information is denoted by $F\left(S_{e}\right)=1$ and the nontransmission of safety-signifying information is denoted by $F\left(S_{e}\right)$ $=0$, the production of the safety confirmation-based output energy $U$ is given by

$$
S_{c}(t) \geq F\left(S_{e}\right) \geq U \text {. }
$$

Equation ( 3 ) means that the function $F\left(S_{e}\right)$ is interposed between the prediction of the safety $S_{c}(t)$ and the resultant production of the output energy $U$. It is indicated by the unate relation $\left[S_{c}(t) \geq U\right]$ that the energy $U=1$ for performing hazardous work should not be produced when it is not safe $\left[S_{c}(t)=0\right]$.

Equation (3) physically involves such a time interval that the information $S_{c}(t)$ is not transmitted to the information transmitting function $F\left(S_{e}\right)$. For example, the energy sampled by a physical sensor as the safety-signifying information $S_{c}(t)=1$ is normally very minute, while the output energy $U$ accompanying hazard is very large. The function $F\left(S_{e}\right)$ thus has an amplifying function, and the amplifying function requires an external source of energy, electrical or mechanical. Unless the external energy is supplied, the output energy $U$ is not produced.

If there is an error in the information-transmitting function $F\left(S_{e}\right)$ and if the operating state of the means $F\left(S_{e}\right)$ is denoted by the binary logic variable $F^{*}$ (logic 1 for the normal state and logic 0 for the faulty state), the function $F\left(S_{e}\right)$ expressed by Eq. (1) must be represented by the following equation ( $A$ logic variable that denotes the operating state of a device is marked with an asterisk, and the normal state and faulty state of the device are denoted by logic 1 and 0 , respectively):

$$
U=F\left(S_{e}\right) \cdot F^{*} \text {. }
$$

Equation ( 4 ) means that the output $U=F\left(S_{e}\right)$ is produced when the function $F\left(S_{e}\right)$ is normal, and that the output $U=0$ is produced when the function $F\left(S_{e}\right)$ is faulty. The energy-transmitting means expressed by Eq. ( 3 ) is called the fail-safe energy-transmitting means.

The information-transmitting means expressed by Eq. ( 3 ) calls for a means for sampling the safetysignifying information $S_{c}(t)$, a means for producing

Table 1 Unate relation $\left[S_{c}(t) \geq U\right]$

\begin{tabular}{c|c}
\hline$S c(t)$ & $U$ \\
\hline 1 & 1 \\
1 & 0 \\
0 & 0 \\
\hline
\end{tabular}


output energy based on Eq. (4) (output-energyproducing means), and sometimes a means for forcibly interrupting the already produced output energy $u(U=1) \quad$ (forcible - output - energy - interrupting means) because the output energy $u(U=1)$ has inertia. The output-energy-producing means involves a process in which the accumulated energy is consumed or dissipated as heat according to the entropy increasing law when the output energy $U=1$, based on Eq. (4), goes $U=0$ due to input energy interruption. Since this process is not fast enough, the forcible output-energy-interrupting means requires a brake for example. The energy-transmitting means discussed in this paper transmits the sampled safety information and has a means for producing output energy from the safety information. The output-energyproducing means involves a means for forcibly interrupting the output-energy-produced when it is hazardous. The output-energy-transmitting means has the error $U=0$ in the output energy.

\section{Logic Model of Safety Confirmation-Type Energy-Transmitting System}

\section{1 Logic construction of energy transmission in a simple machine}

Control, according to which hazardous work is performed, based on the condition of safety, is defined as safety control. The energy-transmitting system in which the supplied energy is based on the condition of safety is discussed here using a simple machine.

The control system of a simple load lifting machine is shown in Fig. 1. When the switch $S w$ is closed, the energy $e$ is supplied from the energy source to the motor $M$. The rotating output of the motor $M$ is transmitted to the pulley, which in turn lifts the load. In this operation, the switch $S w$ should be closed, based on the safety condition that no person should be present beneath the lifted load (indicated by the energy $h)$. If $\boldsymbol{Z}(e, h)$ is the energy-transmitting means composed of the switch, electric wire, motor, belt, and wire rope, the production of the output energy $u$ of the lifting machine can be represented by the block diagram of Fig.2(a). The energy-transmitting means shown in Fig. 2(a) is such that no energy is

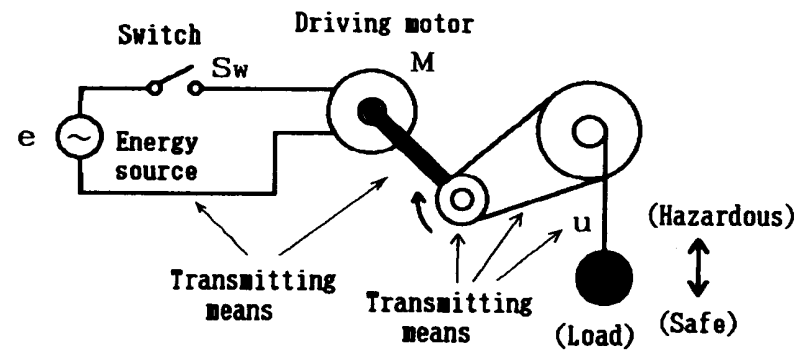

Fig. 1 Control model of load lifting machine transmitted when its components, such as the electric wire, motor, shaft, belt, or wire rope, have failed (or broken). If the input energy $e$ is denoted by the binary logic variable $E \in(1,0)$ (logic 1 for the production of the input energy $e$ and logic 0 for the non-production of the input energy $e$ ), the output energy $u$ is denoted by the binary logic variable $U \in\{1,0\}$ (logic 1 for the production of the output energy $u$ and logic 0 for the non-production of the output energy $u$ ). If the operating state of the energy-transmitting means $\boldsymbol{Z}(e, h)$ is denoted by the binary logic variable (state variable) $Z^{*} \in(1,0)$, then the following logic equation can hold between the input energy $e$ and output energy $u$ :

$$
U=Z^{*} \cdot E \text {. }
$$

Equation ( 5 ) means that the output energy $u$ is produced only when the input energy $e$ is supplied ( $E$ $=1)$ and the energy-transmitting means is normal $\left(Z^{*}\right.$ =1). $E \in\{1,0\}$ can be taken to correspond one-to-one with the normal or faulty state of the energy source. If $E^{*}$ is the operating state of the energy source, Eq.

( 5 ) can be rewritten as follows:

$$
U=Z^{*} \cdot E^{*} \text {. }
$$

Equation (6) means that the supply of the output energy $u$ depends on the energy source $\left(E^{*}\right)$ and transmitting means $\left(Z^{*}\right)$. The output energy $u$ is supplied when the condition of safety is satisfied and is not supplied when the condition of safety is not satisfied. If the work environment in which the output energy $u$ is produced is divided into the safe state and hazardous state; and if the state of the environment is denoted by the binary logic variable $S_{e} \in\{1,0\}$ (logic 1 for the safety state and logic 0 for the hazardous state), Eq. (6) must be represented by

$$
U=S_{e} \cdot E^{*} \cdot Z^{*} \text {. }
$$

Equation ( 7 ) means that the output energy $u$ is produced $(U=1)$ only when the work environment is safe $\left(S_{e}=1\right)$ and the energy transmitting and supplying systems are normal $\left(Z^{*}=1, E^{*}=1\right)$ and indicates that the production of the output energy $u$ accompanies the safety information $S_{e}$.

Equation ( 7 ) indicates that the energy-transmitting means $Z(e, h)$ shown in Fig. $2(\mathrm{a})$ is an AND gate that performs the AND operation $S_{e} \cdot E^{*}$. The energy-transmitting means $Z(e, h)$ is represented by an AND gate in Fig. 2 (the AND value is $S_{e} \cdot E$ because $E^{*}=E$ ).

It is assumed here that the energy transmitting system has an internal energy source as shown in Fig. 2 (a) and is a device that has the function [indicated by $Z_{e}(h)$ ] of producing the output $u$ for the safety condition $h$. The operating state of the device is denoted by $Z_{e}^{*} \in\{1,0\}$. The device must guarantee $Z_{e}^{*}$ $=E^{*} \cdot Z^{*}=0$, for example, when the energy source fails and when the energy transmitting system fails 
(the device must not produce its internal energy as the output $u$ when it fails). This requirement must be represented by the following logic expression:

$$
U=S_{e} \cdot Z_{e}^{*} \text {. }
$$

If Eq. ( 8 ) is applied to general equipment, the form of energy supply involved is not clear and is extremely complicated (when electric circuity is included, the energy source can assume a great variety of forms). The logic relationship between the safety condition $S_{e}$ and energy supply $E$ is not clearly represented either. The energy source and energy - transmitting means $Z(e, h)$ are handled as separate systems, as expressed by Eq. 7 ).

\subsection{Energy-transmitting means with external energy source}

In Eq. ( 7 ), external energy supply is handled as the logic variable $E$ (or information) as shown in Fig. 2 (b). The production of output is represented by the following logic product :

$$
U=S_{e} \odot E \text {. }
$$

The following logic relations must hold between $S_{e}$ and $E$ :

Condition (1): $S_{e} \geq U, E \geq U$.

Namely, $U=1$ must not be produced when $S_{e}=0$ or $E$ $=0$.

Condition ( 2 ): The AND operation expressed by Eq. (9) must not produce the output $U=1$ from the condition of either $S_{c}=1$ or $E=1$ even if the AND gate is faulty. The logic product with this characteristic is called the fail-safe logic product and is represented by the logic product symbol $\odot$ as expressed by Eq. ( 9 ).

The meaning of the logic product $A \odot B$ expressed by the symbol $\odot$ is clarified here.

The fail-safe energy transmission defined by Eq. (4) requires such a characteristic that the output $U$ $=0$ is produced when the energy-transmitting means $F\left(S_{e}\right)$ fails $\left(F^{*}=0\right)$. The fail-safe operation defined by the logic product $A \odot B$ demands that the operation

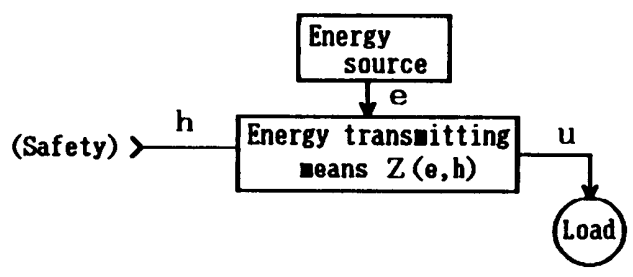

(a) Energy-transmitting system for load lifting machine of Fig. 1

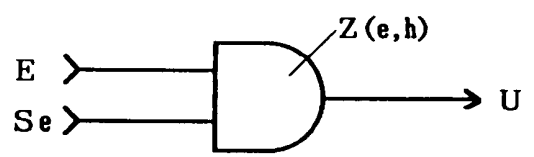

(b) Logic representation of energy transmission

Fig. 2 Energy transmission in safety control system output $F(A, B)=0$ must be produced when the AND gate fails $\left(F^{*}=0\right)$. In addition, the output $F(A, B)=1$ must not be produced (or no output must be produced from either operation input $A$ or $B$ alone) when the AND gate fails and the logic product $A \cdot B$ does not hold. In this case, the operating state of the AND gate is denoted by the binary logic variable $F^{*} \in\{1,0\}$, and the operation output $F(A, B)$ is denoted by $F(A, B)$ $=(A \odot B) \cdot F^{*}$.

$S_{e} \geq U$ in the above condition (1) means that when the safety information $S_{e}=0$ is available in Fig. 2 (b), the switch $S_{w}$ must not be erroneously closed in Fig. 1. The condition (2) means that such an event must not occur that $U=1$ is produced when $E=1$ (energy supply is normal or $E^{*}=1$ ), although the contacts of the switch $S_{w}$ are welded and $S_{e}=0$, or that the human operator provides another energy source and produces $U=1$ when $E=0$ (the energy source is faulty or $E^{*}=0$ ), although $S_{e}=0$.

The energy transmitting system with control performed based on safety information by using external energy is fail-safe when the above-mentioned conditions (1) and (2) are both satisfied and is not fail-safe when either condition (1) or (2) is not satisfied.

In Fig. 2(a), safety is predicted by the human operator or safety information is sampled by the human operator. In this case, the safety information $S_{e}$ is visually sampled by the operator as small energy and is amplified by him into such energy as to operate the switch $S_{w}$. The output energy to operate the switch $S_{w}$ as expressed by Eq. ( 7 ) must be produced between the safety information $S_{e}$ to be sampled and the energy source possessed by the operator (this energy source is denoted by $E_{m}$ ). The operator makes a mistake, however. The function of the operator with the energy source is denoted by $Z_{m}(h)$ in accordance with Fig. 3. The state of the operator is denoted by the binary logic variable $Z_{m}^{*}$ (logic 1 for the normal state and logic 0 for the abnormal state). When the operator is not normal, he may or may not close the switch $S_{w}$. This situation is denoted by the binary logic variable $E_{m} \in\{1,0\}$ (there is assumed to be only one operator involved) with logic 1 for the supply of energy and logic 0 for supply of no energy. The operator's switch operation $H_{m}$ is thus given by

$$
H_{m}=S_{e} \cdot Z_{m}^{*} \vee E_{m} \cdot \bar{Z}_{m}^{*}
$$

Equation (11) means $H_{m}=S_{e}$ when the operator is normal $\left(Z_{m}^{*}=1, \bar{Z}_{m}^{*}=0\right)$ and $H_{m}=E_{m}$ when the opera-

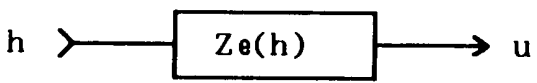

Fig. 3 Energy-transmitting system with internal energy source 
tor is not normal $\left(Z_{m}^{*}=0, \bar{Z}_{m}^{*}=1\right)$.

The system that transmits safety information in a fail-safe manner by using external energy can be expressed by Eq. (7) [Fig. 2(b)]. The system is represented by the following equation as amplifier with the external energy $U$ :

$$
U=E^{*}\left(S_{e}\right) \text {. }
$$

Equation (12) means that the safety information $S_{e}$ is transmitted by the energy transmitting system with the external energy $E$.

\section{Safety Information-Transmitting System with External Energy}

\section{1 Fail-safe logic operations}

Here is considered an energy-transmitting system composed of elements that transmit $n$ items of safety information $S_{e i}(i=1,2, \cdots, n)$ and produce $n$ items of output $U_{0 i}=E_{i}^{*}\left(S_{e i}\right)(i=1,2, \cdots, n)$. The output $U_{0}$ composed of logic products and sums is defined by

$$
\begin{aligned}
& U_{0}=E_{1}^{*}\left(S_{e 1}\right) \odot E_{2}^{*}\left(S_{e 2}\right) \odot \cdots \odot E_{n}^{*}\left(S_{e n}\right) . \\
& U_{0}=E_{1}^{*}\left(S_{e 1}\right) \oslash E_{2}^{*}\left(S_{e 2}\right) \oslash \cdots \otimes E_{n}^{*}\left(S_{e n}\right) .
\end{aligned}
$$

Equation (13) indicates $n$ fail-safe logic products defined as in the conditions (1) and (2) in Eq. (9). The symbol $\Theta$ in Eq. (14) indicates a logic sum. The logic sum refers to the failure of an element that outputs $U_{0 i}=E_{i}^{*}\left(S_{e i}\right)(i=1,2,-, n)$ among $n$ items of output or to the transmission of output that is not $U_{0 i}$ $=0$ when $S_{e i}=0$ (it is not safe).

Multiple energy sources are utilized in a general energy transmission system. An example is the transmission of multiple items of safety information by multiple electromagnetic relays, each with a different energy source. The electromagnetic relays have the amplifying function of converting small current to energize the coil into large contact current.

Equation (13) can be represented in Fig. 4 as an energy transmission system with electromagnetic relays $(n=2)$, for example. In the energy transmission system of Fig. $4, S_{e 1}$ and $S_{e 2}$ are safety information given at the contacts from outside; $C R_{1}$ and $C R_{2}$ are electromagnetic relays, each of which is composed of an electromagnetic coil and mechanical contacts ; and $M$ is a motor driven by the result $U$ of logic operation through the relay contacts. The driving energy (cur-

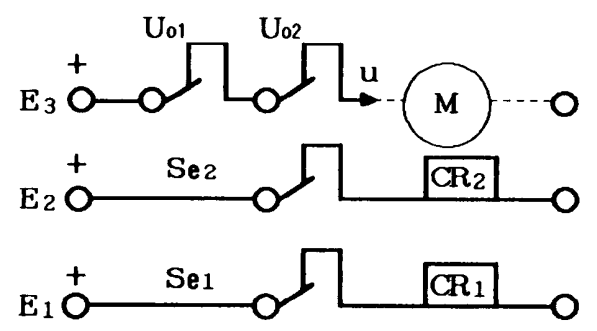

Fig. 4 Energy transmission by logic product rent) $U$ of the motor $M$ is produced by the logic product $U_{01} \cdot U_{02}$ of relay outputs with respect to the contact inputs $S_{e 1}$ and $S_{e 2}$. It is assumed here that $S_{e 1}$, $S_{e 2}, E_{1}, E_{2}, E_{3}, U_{01}$, and $U_{02}$ can be handled as binary logic variables. If the electromagnetic relays are constructed in a fail-safe manner as described later and their contacts do not weld, the energy transmission system of Fig. 4 can be described by

$$
\begin{aligned}
& U=\left\{\left(S_{e 1} \odot E_{1} \cdot Z_{1}^{*}\right) \odot\left(S_{e 2} \odot E_{2} \cdot Z_{2}^{*}\right\} \odot E_{3} \cdot Z_{3}^{*}\right. \\
& \quad \therefore \quad U=U_{0} \odot E_{3} \cdot Z_{3}^{*},
\end{aligned}
$$

where $U_{0}=U_{01} \odot U_{02}$. That is,

$$
\begin{aligned}
U_{0} & =\left(S_{e 1} \odot E_{1} \cdot Z_{1}^{*}\right) \odot\left(S_{e 2} \odot E_{2} \cdot Z_{1}^{*}\right) \\
& =E_{1}^{*}\left(S_{e 1}\right) \odot E_{1}^{*}\left(S_{e 2}\right),
\end{aligned}
$$

where $Z_{1}^{*}, Z_{2}^{*}$, and $Z_{3}^{*}$ are the binary logic variables that indicate the operating state of an energy-transmitting means composed of electric conductors, coils, and mechanical contacts (or relays). Especially, $Z_{3}^{*}$ indicates the operating state of the electric conductor that produces the output $U$.

If the energy sources $E_{1}$ and $E_{2}$ are common ( $E_{1}$ $=E_{2}$ ) in Fig. 4, Eq. (16) can be rewritten as follows:

$$
\begin{aligned}
U_{0} & =\left(S_{e 1} \cdot Z_{1}^{*}\right) \odot\left(S_{e 2} \cdot Z_{2}^{*}\right) \odot E_{1} \\
& =S_{e 1} \odot S_{e 2} \odot E_{1} \cdot Z_{1}^{*} \cdot Z_{2}^{*} \\
& =S_{e 1} \odot S_{e 2} \odot E_{1} \cdot Z_{12}^{*} \\
& =E_{1}^{*}\left(S_{e 1} \odot S_{e 2}\right),
\end{aligned}
$$

where $Z_{12}^{*}=Z_{1}^{*} \cdot Z_{2}^{*}$. This indicates the operating state of the energy transmission system composed of the relays $C_{1}$ and $C R_{2}$, and signifies that the output $U_{0}$ is not produced when either $\mathrm{CR}_{1}$ or $\mathrm{CR}_{2}$ is faulty.

In $\left(S_{e 1} \cdot Z_{1}^{*}\right) \odot\left(S_{e 2} \cdot Z_{2}^{*}\right) \odot E_{1}$ of Eq. $(17),\left(S_{e 2} \cdot Z_{2}^{*}\right) \odot$ $E_{1}$, for example, must be such a logic product operation that $Z_{2}^{*}=1$ or $Z_{2}^{*}=0$ is produced as information and that $Z_{2}^{*} E_{1}$ is fail-safe. That is, $Z_{2}^{*}$ indicates the operating state of an energy-transmitting element, and the logic product of $Z_{2}^{*}$ with the energy $E_{1}$, or $Z_{2}^{*} \cdot E_{1}$, does not indicate a fail-safe logic operation. Since $Z_{2}^{*} \in\{1,0\}$ is produced as a signal here, the failsafe logic operation $Z_{2}^{*} \odot E_{1}$ is made possible.

Each of the terms enclosed in parentheses in Eq. (17) indicates a logic operation in the energy-transmitting system of Fig. 4. If multiple items of binary input information $S_{e 1}, S_{e 2}, \cdots, S_{e n} \in\{1,0\}$ are denoted by the logic variable $X$, that is, $X=\left\{S_{e 1}, S_{e 2}, \cdots, S_{e n}\right\}$, the function of the energy-transmitting system that performs fail-safe processing on the logic variable $X$ is denoted by $f(X)$, the operating state of the energy -transmitting system is denoted by $f^{*}$, and the energy source (electric energy source) required for the processing is denoted by $E$, the output $U_{0}^{\prime}$ of the processing can be given by

$$
U_{0}^{\prime}=f(X) \odot E \cdot f^{*}=E^{*}\{f(X)\} .
$$

\subsection{Example of fail-safe logic operation with external energy}

Now an energy-transmitting system in which the 
safety information $S_{e}$ is sequentially transmitted by using multiple energy sources is discussed.

A general processor is shown in Fig. 5. It consists of a sensor [energy-transmitting means $Z\left(e_{1}, h\right)$ ] that physically samples the safety-signifying information (energy) $h$ and produces the amplified output $h^{\prime}$ and of a means $Z\left(e_{2}, h^{\prime}\right)$ that produces the mechanical output energy $u$ only when the output energy $h^{\prime}$ is produced. In Fig. $5, e_{1}$ is the electrical energy source of the sensor $Z\left(e_{1}, h\right)$, and $e_{2}$ is the mechanical energy source of the energy-transmitting means $Z\left(e_{2}, h^{\prime}\right)$. If $h, e_{1}, e_{2}$, and $u$ are similarly denoted by the binary logic variables $S_{3}, E_{1}, E_{2}$, and $U \in\{1,0\}$, respectively, and if the operating state of the energy-transmitting means $Z\left(e_{1}, h\right)$ and $Z\left(e_{2}, h^{\prime}\right)$ is denoted by the binary logic variables $Z$ and $Z \in\{1,0\}$, respectively, the output $U$ is defined by

$$
\begin{aligned}
U & =\left(S_{e} \odot E_{1} \cdot Z_{1}^{*}\right) \odot E_{2} \cdot Z_{2}^{*} \\
& =E_{2}^{*}\left\{E_{1}^{*}\left(S_{e}\right)\right\} .
\end{aligned}
$$

Equation (19) indicates that the safety device shown in Fig. 5 must not produce the output $U=1$ when either the means $Z\left(e_{1}, h\right)$ or $Z\left(e_{2}, h^{\prime}\right)$ fails, and that the external energy $E_{1}=1$ and $E_{2}=1$ must not be produced as $U=1$ when $S_{e}=0$ in the faulty state.

The authors developed a fail-safe AND circuit, called the operational oscillator ${ }^{(4),(5)}$. The fail-safe AND circuit has amplifiers (including photocoupled amplifiers) cascade connected, inspects the amplifiers to see that they are normal, and produces output accordingly. The principle of its construction is shown in Fig. 6, where $A_{1}, A_{2}, \cdots, A_{n}$ are amplifiers, $\mathrm{G}_{e}$ is the inspection signal generator (ac signal generator) for the operating states $A_{1}^{*}, A_{2}^{*}, \cdots, A_{n}^{*} \in\{1,0\}$ of the

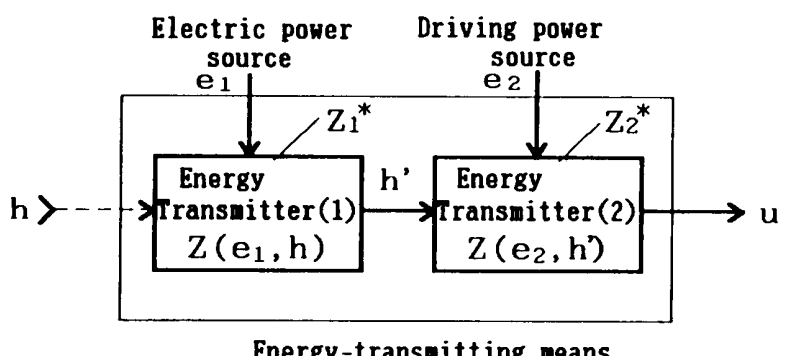

Fig. 5 Safety control system using sensor

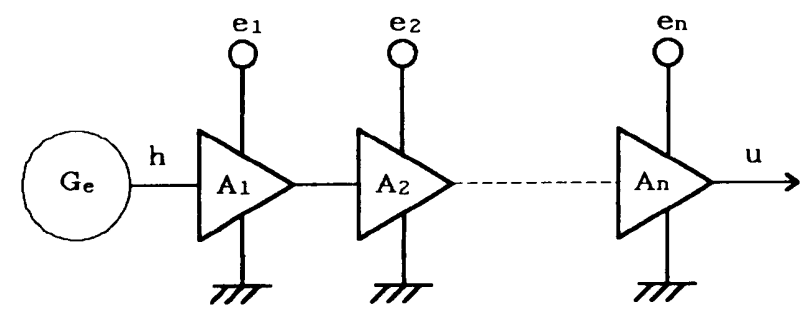

Fig. 6 Fail-safe AND circuit amplifiers $A_{1}, A_{2}, \cdots, A_{n} \cdot e_{1}$ to $e_{n}$ are the electrical energy sources of the amplifiers $A_{1}, A_{2}, \cdots, A_{n}$ and are input signals for logic operations here. In other words, the output $u$; is produced only when the input signals $e_{1}, e_{2}, \cdots, e_{n}$ are all available. The amplifiers $A_{1}, A_{2}, \cdots$, $A_{n}$ are normal when the inspection signal $h$ is produced as the output $u$, and the output energy $u$ is not produced when any one of the amplifiers is faulty. The production of the output $u$ against the signal $h$ indicates safety (as logic product output of the input signals $e_{1}, e_{2}, \cdots, e_{n}$ if the input signals $e_{1}, e_{2}, \cdots, e_{n}$ are safety signifying information). If the inspection signal $h$, input signals $e_{1}, e_{2}, \cdots, e_{n}$, and output $u$ are denoted by the binary logic variables $S_{e}, E_{1}, E_{2}, \cdots, E_{n}$, and $U$ $\in\{1,0\}$,

$$
U=\left(S_{e} \odot E_{1} \cdot A_{1}^{*}\right) \odot E_{2} \cdot A_{2}^{*} \odot \cdots \odot E_{n} \cdot A_{n}^{*} .
$$

The inspection signal $h$ is not produced when the inspection signal generator $G_{e}$ is faulty. If the operating state of the inspection signal generator is denoted by the binary logic variable $G_{e}^{*} \in\{1,0\}, G_{e}^{*}=S_{e}$. If the electric energy source inputs $E_{1}, E_{2}, \cdots, E_{n}$ are taken as logic inputs, the amplifiers of Fig. 6 are represented as a fail-safe circuit by

$$
U=E_{1} \odot E_{2} \odot \cdots \odot E_{n} \cdot A_{2}^{*},
$$

where

$$
A_{z}^{*}=G_{e}^{*} \cdot A_{1}^{*} \cdot A_{2}^{*} \cdots A_{n}^{*},
$$

where $A_{z}^{*}$ is $A_{z}^{*}=0$ when either the inspection signal generator $G_{e}$ or amplifiers are faulty.

\section{Output Producing Elements}

The energy source must be constructed so that the output energy $U$ is not produced as $U=1$ when the safety information $S_{e}$ expressed by Eq. ( 7 ) is $S_{e}=0$. Usually, the safety information $S_{e}$ is physically produced with small energy as sensor input. There is an energy transmission process by amplification as shown in Figs. 4 and 5. Electromagnetic relays and electronic circuits are utilized for this energy transmission.

\subsection{Unate energy transmission by electronic circuit}

Normally, output from an electronic circuit is characteristic in that it is directly produced from an amplifying electric energy source in the event of failure or it goes zero. This is called the asymmetrical error characteristic. Consider an amplifier circuit composed of the resistor $\mathrm{R}$ and transistor $\mathrm{Q}$ as shown in Fig. 7. When the collector $C$ of the transistor $Q$ is broken, the electric energy source $e_{1}$ is outputted to the terminal a even if the input voltage $(h=1)$ is not applied at the base $B$ of the transistor $Q$. When the collector $\mathrm{C}$ and emitter $\mathrm{E}$ of the transistor $\mathrm{Q}$ are short -circuited, the output goes zero (or exhibits the asymmetrical error characteristic). Like the output signal 
(energy) $u$ in Fig. 6, for example, the signal $h$ inputted to the base $B$ of the transistor $Q$ is an ac signal (the safety information $h$ goes 1 when the ac signal is available and goes 0 when the ac signal is not available). The amplified output signal (designated $u$ again) is produced superimposed on the electric energy source $e_{1}$ supplied from outside with a voltage doubler rectifier circuit composed of the capacitors $C_{1}$ and $C_{2}$ and diodes $D_{1}$ and $D_{2}$. The output $u$ at the terminals $P_{1}$ and $P_{2}$ is produced in the presence of the safety information $h(\mathrm{ac})$ and is not produced in the absence of the safety information $h$ (ac). In this way, the amplified output is produced superimposed on the electric energy source $e_{1}$ supplied for amplification. The output $u$ is produced at a higher level than that of output from the external environment, including the electric energy source. No output at a level higher than that of the electric energy source $e_{1}$ is produced at the output terminal $P_{2}$ in the absence of the input $h$ when any circuit element is faulty. (The latter processing is called processing outside of the electric energy source frame $\mathrm{e}^{(6)}$.) If the input $h(\mathrm{ac}) \in\{1,0\}$ is denoted by the binary logic variable $S_{e}$, and the operating state of the circuit, electric energy source $e_{1}$, and output $u$ are denoted by the binary logic variables $Z^{*}, E^{*}$, and $U \in\{1,0\}$, respectively, the output $U$ can be expressed by Eq. $(7)$.

\section{2 Logic of output interruption (brake)}

When the input information or energy supplied from outside disappears in the energy-transmitting system shown in Fig. 2(a), 4, or 7, the output $u$ is dissipated according to the entropy increasing law (or as heat). In reality, however, the output energy may not be properly interrupted by heat dissipation. In that

Table 2 Logic relation for output interruption

\begin{tabular}{c|c|c}
\hline $\mathrm{X}$ & $\mathrm{Y}$ & $\mathrm{Z}$ \\
\hline 0 & 0 & 0 \\
\hline 1 & 0 & 0 \\
\hline 0 & 1 & 1 \\
\hline 1 & 1 & 0 \\
\hline
\end{tabular}

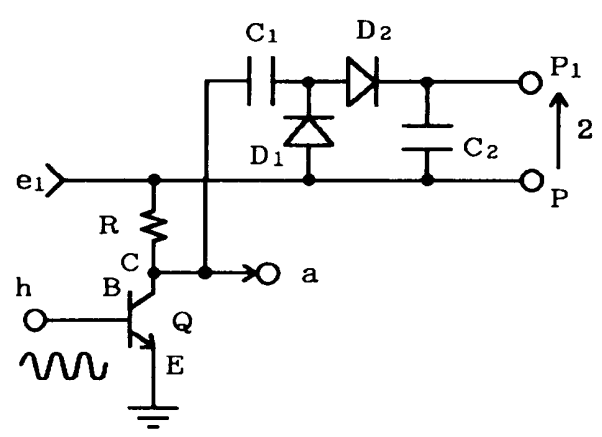

Fig. 7 Realization of $V=S_{e} \odot E_{1} \cdot Z^{*}$ in electronic circuit case, the output energy must be forcibly interrupted.

Let $\psi$ be the driving energy of the machine, $\omega$ be the machine output actually produced by the driving energy, and $x$ be the energy required to forcibly stop the machine output $\omega$. In this case, $x>\phi$. As far as the magnitude relationship between $\omega$ and $x$ is concerned, it is necessary to consider the output energy $\omega$, including the energy accumulated during the operation of the machine, and the stopping energy $x$ that allows for energy dissipation during the forcible stop. Here we simply set $x>\omega$. There is a trancient phase between start and stop, but the logic conditions of start and stop are ignored here.

The way the output energy $\omega$ is produced and interrupted is logically discussed here. The driving energy $\psi$, the output energy $\omega$, and stopping energy $x$ are denoted by the binary logic variables $Y, Z$, and $X$, respectively, with logic 1 for the presence of each energy and logic 0 for the absence of each energy. $Z$ $=1$ indicates that the machine is in operation, and $Z$ $=0$ indicates that the machine is stopped. The logic relations shown in Table 2 should at least hold among $X, Y$, and $Z . X=1$ and $Y=1$ in Table 2 indicate that the stopping energy and driving energy are applied at the same time. $Z=0$ refers to the braking energy and means that the machine should be stopped with priority given to $X=1$. The energy required to assure $x>$ $\psi$ is large (as an emergency stopping means, for example). In this case, the driving energy is interrupted $(\phi=0)$. The condition of $X=1, Y=1$ is interpreted as that of error. The condition of $X=0, Y=0$ is what is called the loose condition and is likely to produce $Z=1$ when external energy (a kind of noise) is applied due to some error. For this reason, the condition of $X=0, Y=0$ is avoided, and the machine is kept in the condition of $X=1, X=0$ when it is stopped. The logic construction enclosed by the dotted line in Table 2 applies when the machine is in operation.

The machine must go through the process of ( $X$ $=1, Y=0) \rightarrow(X=0, Y=0) \rightarrow(X=0, Y=1)$ to enter from the stop condition of $X=1, Y=0$ to the operating condition of $X=0, Y=1$ or return from the operating condition of $X=0, Y=1$ to the stop condition of $X=1, Y=0$. The mutually negative forms of energy transmission, or $X=1 \rightarrow X=0$ and $Y=0 \rightarrow Y=1$, are required between the stop condition of $X=1, Y=0$ and the operating condition of $X=0, Y=1 . Y \geq Z$, but not $X \geq Z^{(7)}$. If the driving energy $Y$ is taken as the output $U$ based on the permission $S_{e}$ on the condition of $X=0$, the system becomes a heat dissipation system. If the driving energy $U$ is given as a brake system and the stopping energy $X$ is supplied based on the permission $S_{e}$, the system becomes a normally 


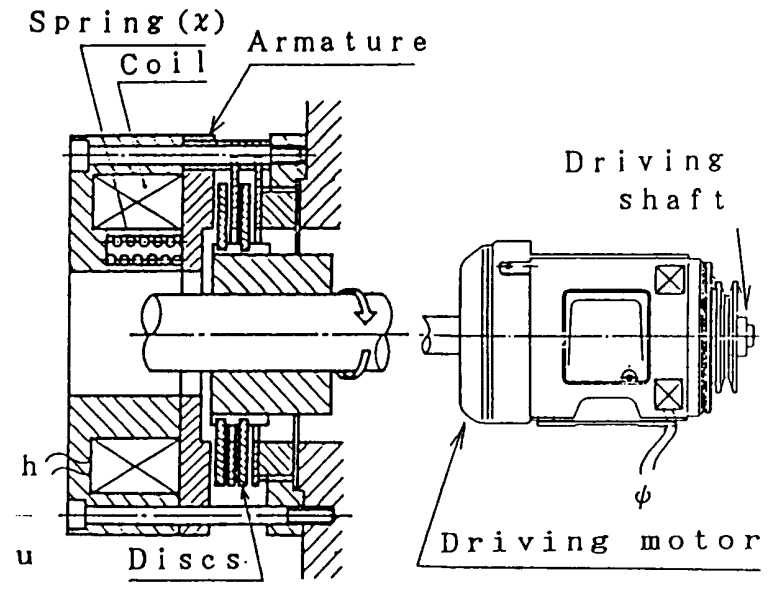

Fig. 8 Electromagnetic brake (normally closed)

open brake system. A NOT relation is required between the input permission $S_{e}$ and the stopping energy $x$ (that is, $X=\bar{S}_{e}$ ), and energy transmission is not unate (nor fail-safe). An electromagnetic brake of normally closed construction is thus used as shown in Fig. 8.

The electromagnetic brake of Fig. 8 is normally engaged by the springs (the spring energy is denoted by $x$ ). The motor produces the rotating output $\omega$ from the driving energy $\phi$ only when the energy $u$ is supplied to disengage the brake. The logic value $X=0$ is accomplished when the brake-disengaging energy $u$ is superimposed on the spring energy $x$ at the time of stop, and $Y \geq Z$ in logic terms. If the brake-disengaging energy is denoted by the binary logic variable $S_{e}$ (logic 1 for the presence of the energy and logic 0 for the absence of the energy), $S_{e} \geq Z$. The electromag. netic brake must be constructed so that the spring energy $x$ is not consumed as energy output $(x \neq 0)$. Table 3 is a truth table that gives the logic relations between the stopping input $S_{e}$ and driving input $Y$. The output $Z$ is produced only when $S_{e}=Y=1$ and is given by

$$
Z=S_{e} \cdot Y \text {. }
$$

Since the stopping input $S_{e}$ is based on the start permission and the driving input $Y$ is based on the start command, the electromagnetic brake of Fig. 8 comprises an interlock.

\section{3 Electromagnetic relays}

A device equiped with an amplifying function is indispensable if large energy is to be obtained for energy interruption. Figure 9 shows an electromagnetic relay that interrupts a large output (current) $u$ by a small input. The logic relation $S_{e} \geq U$ must be maintained between the input energy $h$ and output energy $u$. As in the electromagnetic brake of Fig. 8, the spring energy $\chi$ (equivalent to $X=1$ in Table 3 ) is required to securely keep off the contacts on the
Table 3 Condition $Z$ of machine operation

\begin{tabular}{c|c|c}
\hline$S D \backslash Y$ & 1 & 0 \\
\hline 1 & 1 & 0 \\
\hline 0 & 0 & 0 \\
\hline
\end{tabular}

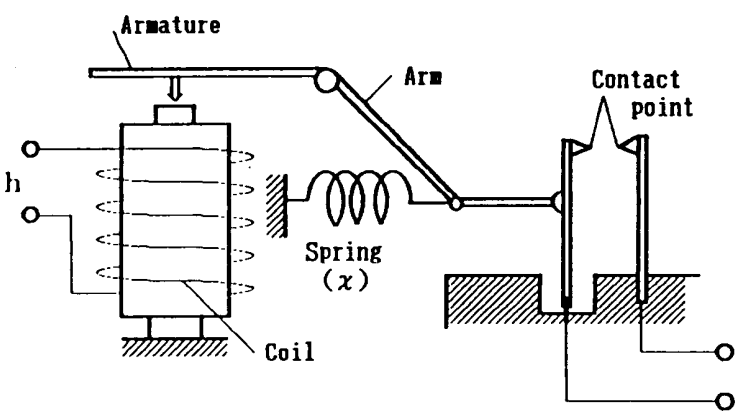

Fig. 9 Electromagnetic relay

condition that the spring does not fail $(X \neq 0)$. If the output current $u$ is allowed to flow through the spring, it is not produced when the spring breaks. The electromagnetic coil supplies the energy that is superimposed on the spring energy to close the contacts.

\section{Conclusions}

The principles of safety were presented, and the unateness of safety information transmission that is inevitably required in the safety confirmation process was clarified as the logic of the amplification process, including the machine system. The safety information to be sampled by a sensor must be amplified as energy that is outputted only when it is safe. Energy must be supplied from outside for the amplification. For this reason, the logic relationship of safety information to be sampled as energy was described by an AND gate model. The applicability of the model to concrete fail -safe computing operations was indicated. It was also shown that a fail-safe output energy-producing means can realize the model if external energy is supplied superimposed on the safety information.

\section{References}

(1) Sugimoto, N., Futsuhara, K. and Mukaidono, M., A Study from a Control View Point (Safety Control), Proc. of 6th Lecture Meeting of Robotics Soc. of Jpn., (in Japanese), 2601, (1988), p. 359.

(2) Futsuhara, K., Sugimoto, N. and Mukaidono, M., Fail-safe Technology, Proc. of 5th Lecture Meeting of Robotics Soc. of Jpn., (in Japanese), 1301, (1987), p. 71.

(3) Futsuhara, K. and Sugimoto, N., A Logical Consideration of the Safety Operation System of the Safety Confirmation Type, Trans. Jpn. Soc. Mech. Eng., (in Japanese), Vol. 56, No. 529, C (1990), p. 2378. 
(4) Tsuchiya, S., Studies on Fail-safe Logical Systems, Research of the Electrotechnical Laboratory, (in Japanese), No. 695 (1969), p. 19.

( 5 ) Futsuhara, K., Sugimoto, N. and Mukaidono, M., A Trial Manufacture of Safety Device for Protection Noise-induced Accidents in Computer Controlled Machine, Reports of Soc. Elec. Infor. Com., (in Japanese), EMCJ 89-55 (1989), p. 139.

(6) Futsuhara, K. and Mukaidono, M., Properties and Construction Methods of Fail-safe Train Sensor using Short-Circuit between Rails, Trans. Inst. Elec. Eng. Jpn., (in Japanese), Vol. 107, No. 10, C (1988), p. 955.

( 7 ) Sugimoto. N., Kumekawa, S., Fukaya, K., Shimizu, S., Umezaki, S., Ikeda, H., Houshi, T. and Futsuhara, K., Fundamental Structure of Safety of the Safety Confirmation Type, Trans. Jpn. Soc. Mech. Eng., (in Japanese), Vol. 54, No. 505, C (1988), p. 2284. 